\title{
Autumn MIST 2015
}

\author{
John Coxon and colleagues \\ sum up a Magnetosphere, \\ lonosphere and Solar-Terrestrial \\ meeting that touched on topics \\ across the solar system.
}

\author{
1 Conjugate maps \\ of auroras in the \\ northern and southern \\ hemispheres observed \\ by IMAGE (middle) \\ and DMSP/SSUSI (top/ \\ bottom). A and B label \\ conjugate transpolar \\ arcs moving in both \\ hemispheres. (Jenny \\ Carter)
}

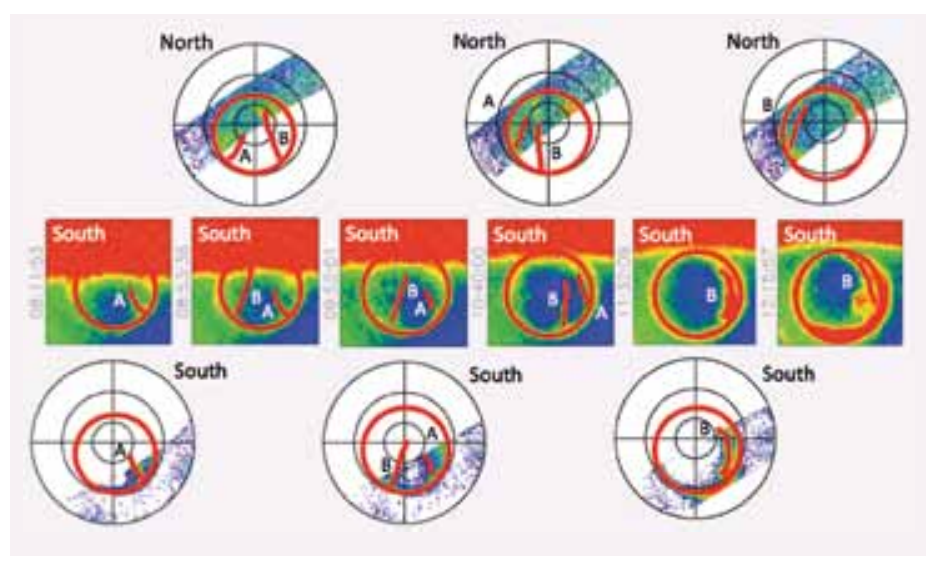

what information they can yield.

The keynote speaker was Betty

Lanchester (formerly head of the Space Environment Physics group, University of Southampton). Her talk focused on multiscale processes of the aurora, using All Sky Imager (ASI) data to show observations of the twin-cell convection pattern and then asking how small-scale motions connect to the observed large-scale flows. Lanchester examined the "black aurora", which consists of structured gaps in the auroral emission as observed from Earth. She showed observations from the Auroral Structure and Kinematics (ASK) instrument (Ashrafi 2007) in support of the theory that the scattering of energetic particles into the loss cone is suppressed, resulting in high-energy gaps in the particle distribution. However, Lanchester pointed out that the cause of this suppression is an open question. ASK observations were presented which showed pulsations in the same region as the black aurora, together with Reimei satellite data that showed the signature of black aurora coincident with pulsating aurora. More ASK observations, this time of flickering aurora, showed an inverse relationship between frequency and energy of the flickering. This is thought to arise from electromagnetic ion acoustic waves transferring energy to electrons via Landau damping.

Nadine Kalmoni (Mullard Space Science Laboratory, MSSL), spoke next about small-scale beads of auroral beads - bright spots of aurora lying along the onset arc - and their relation to substorms. She presented results from the THEMIS and MOOSE arrays of ASIs, showing that beads propagate both east and westwards, with the growth of the beads observed to be fastest at low wavenumbers; spatial scales were also found to have an effect on growth rate, consistent with the idea that the shear flow ballooning instability is important in forming the beads. Kalmoni showed

........ that the beads appear to be "Auroras are perhaps the ubiquitous in onset arcs and most beautiful natural concluded with observations suggesting that the electrons are accelerated by waves (Kalmoni et al. 2015)

enny Carter (University of Leicester) discussed auroral arcs extending into the polar cap, building on Carter et al. (2015) by presenting a case study of two such transpolar arcs using IMAGE observations of the southern hemisphere combined with DMSP SSUSI observations of the northern hemisphere. She outlined the Milan model of transpolar arc formation in which a build-up of closed flux results in a transpolar arc. The model predicts that strong azimuthal flows will be seen at the arc's footprint and that the footprint should be mirrored about the midnight meridian. The two arcs in the case study were found to be mirrored in the two hemispheres on either side of midnight (figure 1) and SuperDARN observed strong flows, consistent with the predictions. 
2 A plot of the jovian system in the XY plane showing the regions to which auroral features map to in Jupiter's magnetotail. (Rebecca Gray)
Moving away from Earth, the next talk looked at auroral processes at Saturn, as Joe Kinrade (Lancaster University) presented auroral images from a 2014 Hubble Space Telescope (HST) campaign. The mean emissions showed features of the steadystate aurora, in which the dawn side had strong discrete emission at $20 \mathrm{kR}$ but the dusk side was more diffuse. Current systems arising from planetary period oscillation (PPO) were examined, showing suppression of auroral intensity of up to $40 \mathrm{kR}$, and the UV intensity in 2014 was found to be ordered by both northern and southern PPO phase.

Rebecca Gray (Lancaster University) moved on to Jupiter, providing an overview of the Jovian magnetosphere and the plasma torus of the jovian moon Io, which forms as a result of volcanic eruptions on Io. The plasma moves outwards as a result of centrifugal forces, distorting the magnetic field and resulting in field-aligned currents that drive the main auroral emissions. HST observations for a case study on 11 January 2014 showed a super-rotating polar spot, a bright and broadened main emission region and equatorward emissions associated with injections of hot, sparse plasma. The spot was theorized to be formed deep in the tail (figure 2), and the super-rotation could be due to Dungey-like reconnection-driven inflow. Associated features in Jupiter's hectometric radio emissions (known as $\mathrm{HOM}$ ) and auroral power have been observed prior to the event, in support of this theory.

The final talk of the session was given by Rosie Johnson (Leicester), who examined the mid-to-low latitude region of Jupiter's ionosphere (which maps equatorward of the Io plasma torus). The study aimed to verify whether this region is corotating; some authors have proposed highvelocity flows in this region (with speeds of $\sim 20 \mathrm{~km} \mathrm{~s}^{-1}$ ) based on observations of longitudinal asymmetries in emission
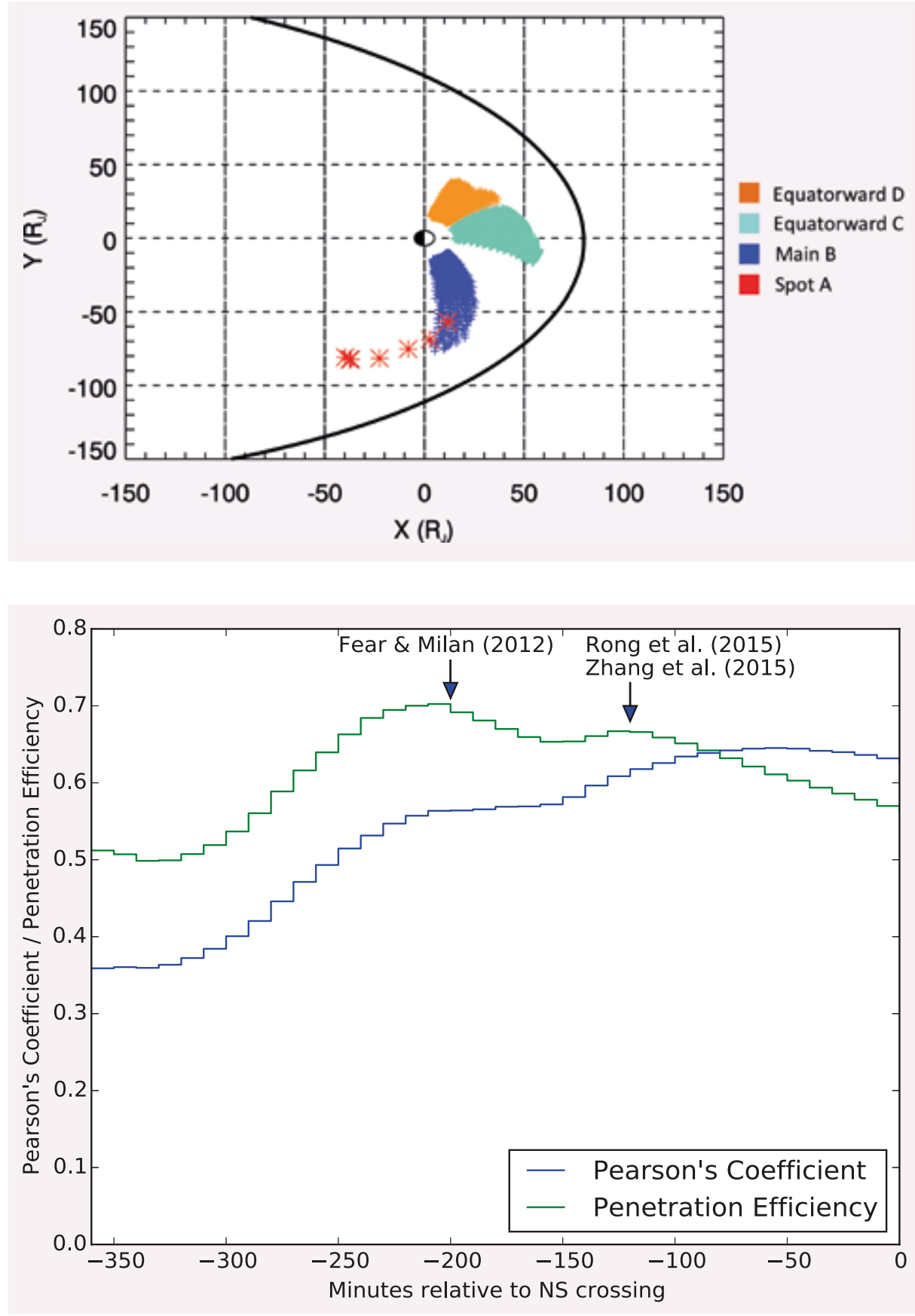

3 The IMF $B_{Y}$ component is lagged by varying lengths of time ( $x$-axis) and then compared to the $B_{Y}$ component in the magnetotail to find the Pearson's correlation coefficient and penetration efficiency. The calculated numbers are plotted on the $y$-axis. (Steve Browett)

intensity. CSHELL observations of $\mathrm{H}_{3}^{+}$ emissions are used to infer these flows and calculate line-of-sight velocities in a jovian rotational reference frame. No longitudinally localized bulge in the expected region was observed and the region was found to be corotating to within $0.5 \mathrm{~km} \mathrm{~s}^{-1}$, indicating that there are no flows of the proposed velocity in this region.

\section{Earth processes}

The T in MIST stands for terrestrial and the second session was preoccupied with Earth-based observations and theory. The first talks focused on substorms and the central magnetotail, before moving to the magnetopause and dayside magnetosphere and finally to the physics of the ionosphere.
Understanding magnetic and plasma processes on Earth is important, partly because space weather and connected phenomena is recognized as a hazard by the Cabinet Office and can have a direct impact on the public. But it is also valuable because understanding these processes at Earth can lead to new insights into similar interactions across the rest of the solar system.

The first talk was given by Sandra Chapman (University of Warwick), who presented innovative work on a network analysis of magnetometers during a substorm using SuperMAG, a network of magnetometers throughout the northern hemisphere. Magnetometers, the nodes in the network, were defined to be "connected" if the cross-correlation between 
4 A plot showing the minimum latitude of the ionospheric trough in degrees against UT, for low Kp (blue), medium Kp (red) and high Kp (green). (James Parker)

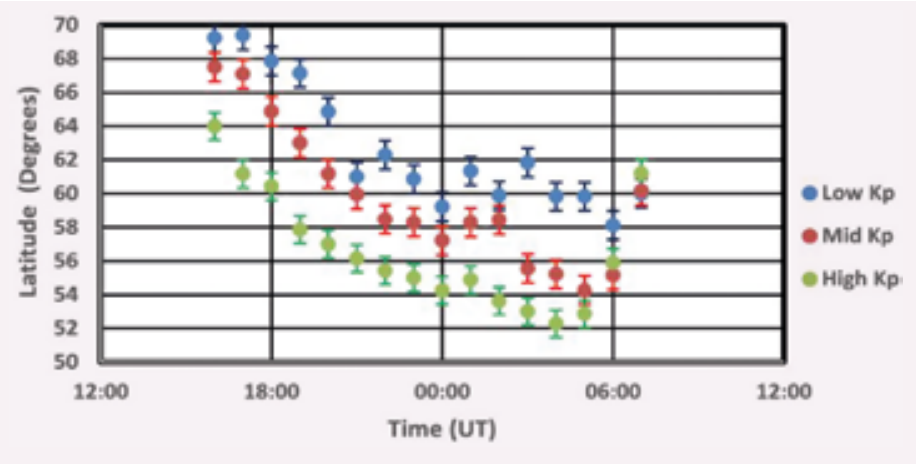

their magnetic field line series exceeded a certain threshold; the number of connections and the number of connected nodes (the size of the network) were analysed throughout the substorm, yielding insight into the spatial structure (Dods et al. 2015).

Colin Forsyth (MSSL) spoke on SOPHIE, his new method of identifying substorm phases using the SuperMAG geomagnetic indices (SML/SMU), which measure the largest magnetic perturbations on the ground caused by westward and eastward currents flowing in the ionosphere. The time derivative of both indices is taken, and negative derivatives in SML above a threshold are identified as substorm expansion phases; positive derivatives in SML are identified as recovery phases. Enhanced convection events are identified and removed by comparison with the time derivative of SMU, and any point not thus identified is highlighted as a potential growth phase.

Forsyth concluded that the SOPHIE expansion phase onsets matched the Frey list better than previous SuperMAG-based lists, and noted that the data are available either as supplementary material to Forsyth et al. (2015) or by request.

Stephen Browett (University of Southampton) moved the session on to the effect of dayside reconnection on the tail, looking at the way in which IMF $\mathbf{B}_{Y}$ affects the value of $\mathbf{B}_{Y}$ during neutral sheet crossings in the magnetotail. Previous work has shown a relation, as it is a consequence of the interaction between the solar wind and the magnetosphere that the IMF $\mathbf{B}_{Y}$ component should "penetrate" into the magnetotail. Browett presented an analysis of the time delay in the correlation between the IMF $\mathbf{B}_{Y}$ component and the $\mathbf{B}_{Y}$ component in the centre of the magnetotail (at low latitudes). Looking at the correlation as a function of time delay, two peaks are found: one at $1 \mathrm{~h} 40 \mathrm{~m}$ and the other at $3 \mathrm{~h}$ (figure 3). Browett suggested that a $\mathbf{B}_{Z}$ effect explained the two different peaks, noting that northward IMF may lead to a slower response from the tail.

Moving away from the central magnetotail, Katie Raymer (Leicester) presented an analysis of crossings of the magnetopause using 17 years of Geotail data. Geotail has an apogee of $30 R_{E}$ (Earth radii) and a perigee of $10 R_{E} \cdot 7770$ magnetopause crossings were identified and compared to a previous model of magnetopause position, showing that the predicted magnetopause positions were further from Earth than those observed. Raymer is working on a model that incorporates the amount of open flux and the strength of the ring current, to attempt to explain these discrepancies.

Tom Elsden (University of St Andrews) presented a model of the dayside magnetosphere, ultra-low frequency (ULF) and magnetohydrodynamic (MHD) waves, with a simple waveguide model which uses a hydromagnetic box approach. This simulation matched the main features of observations made with Cluster and THEMIS, and the authors developed a new boundary condition to effectively drive the model with magnetic pressure, the main source of ULF waves (Elsden \& Wright 2015).

James Parker (Aberystwyth University) took the audience into the ionosphere, looking at the ionospheric trough, an area of low electron density between low and high latitudes that is observed primarily in the F region. Parker outlined a method for modelling the trough, using GPS total electron content (TEC) measurements as inputs to QinetiQ's Electron Density Assimilative Model (EDAM). The trough is seen between mid-afternoon and dawn MLT, with photoionization leading to higher densities at dawn. Results show that the trough moves to lower latitudes during higher geomagnetic activity (figure 4), and this new technique has the potential to allow the trough to be monitored on a dayby-day basis, with more data to be used in the future.

Timothy David (Leicester) talked about the outflow of heavy ions from Earth's ionosphere. Data from the FAST spacecraft alongside the EISCAT Svalbard Radar and the SuperDARN radars were used to explore the dominant mechanisms of ion upwelling and the conditions during which outflow occurs. A local time variation in the flows was observed during summer (with a peak at noon) but not in winter, with higher Kp associated with higher flows. Observations indicated that the upwelling in the winter is velocity driven whereas in the summer it is density driven.

\section{Solar physics and the solar wind}

Although much work in the MIST community focuses on Earth, the ST in the name stands for Solar-Terrestrial and so we also examine phenomena sunward of the magnetopause. The first talk in the final session of the day was given by Mike Lockwood (Reading), who had given the keynote talk at the 2014 Autumn MIST. He returned this year to talk about sunspot numbers, choosing to discuss the different sunspot series and what makes one series more or less believable than another. He talked about analysing the days with no sunspot observations, concluding that this analysis allows for the quality of the observer to be inferred; this is the underpinning of the new series presented by Usoskin et al. (2016).

Chris Chen (Imperial College London) spoke on the definition of plasma instability thresholds using an analysis of multiple particle species, building on Chen et al. (2014) by showing that by considering the dynamics of all major particle populations,

....... the threshold of instability onset can be better defined. He used data from the WIND spacecraft, which spent much of its time in the solar wind, to show that the firehose instability, which was thought to be a
proton instability, has a strong contribution from non-proton components. He also demonstrated that the fluid firehose and mirror instability thresholds constrain the distributions well, which suggests that these instabilities may be present in some form in the solar wind.

Peter Zelina (University of Central Lancashire) talked about solar energetic particles (SEP), which are particles accelerated at the Sun by solar flares and coronal mass ejections. In particular, he focused on the heavy-ion component of the SEP. Combining data from several near-Earth spacecraft, he showed that SEP heavy-ion abundance ratios vary between different $S E P$ events. Heavy-ion SEP data can be used to probe the propagation and acceleration of the particles in the SEP event. The proton $(\mathrm{X} / \mathrm{H})$ ratio decay as a function of time shows a different profile to other heavy-ion ratios.

Georgina Graham (MSSL) presented work examining the solar wind electron velocity distribution. There are three components of the distribution: the core, its surrounding halo and the "strahl", the component that is highly field-aligned. She demonstrated the importance of considering the effect of radial distance from 
5 A plot to show the relationship between the width of the strahl and the energy of its constituent electrons, at 1 au (red crosses) and 5 au (blue asterisks). (Georgie Graham)

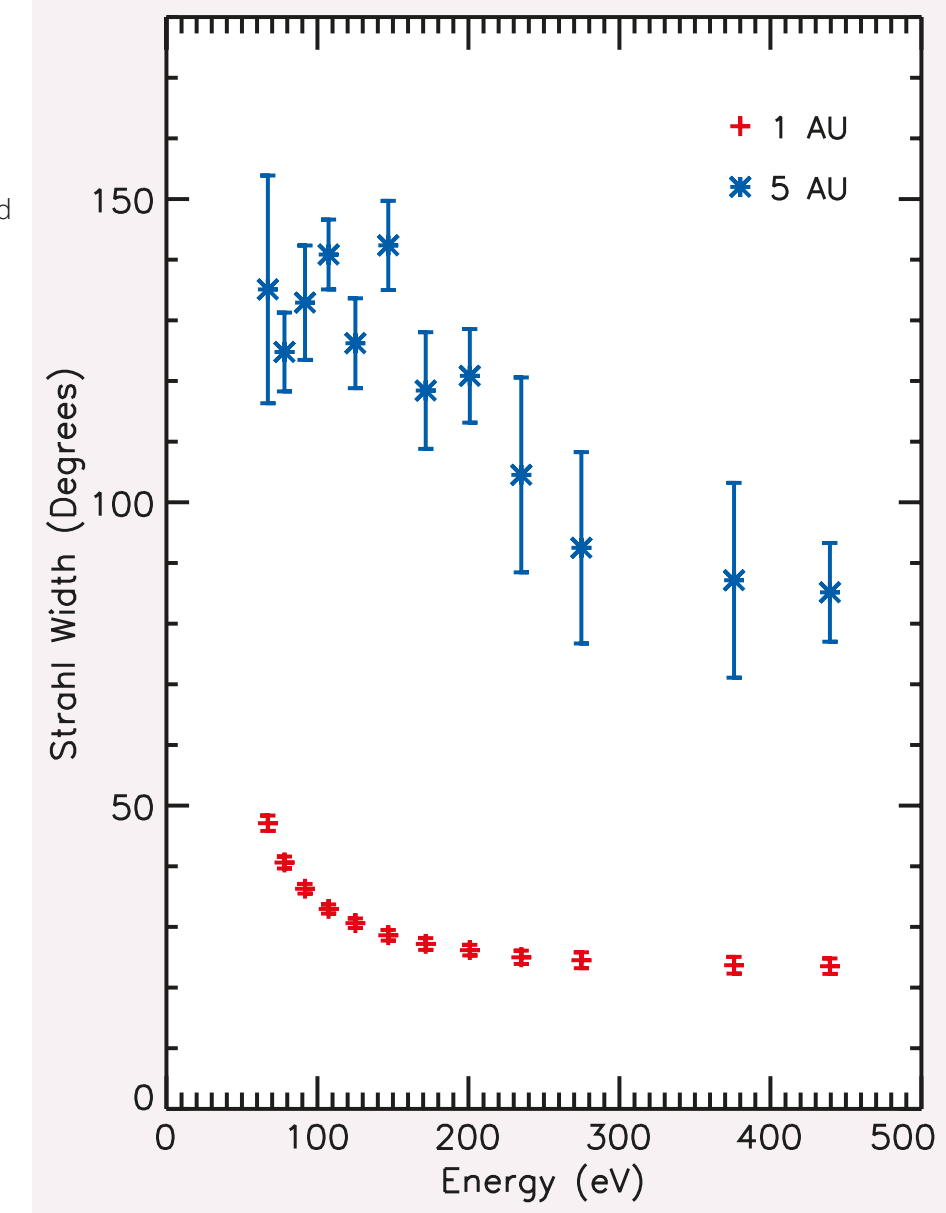

the Sun on the pitch-angle scattering rate (figure 5), and showed Cassini data from its cruise phase that indicate the strahl is most likely to be totally scattered by the time it reaches the distance of Saturn's orbit.

\section{Outer planets}

None of the letters in MIST stand for the outer planets. But the $\mathrm{M}$ and I between them cover the physics of magnetic interactions at the more distant members of our solar system. Much of what we have learned at the Earth informs our studies of the outer planets, whether we are finding similar features or exploring how the systems differ. Starting closest to Earth, Ravindra Desai (MSSL) talked about the plasma environment around Europa, a moon of Jupiter. He explored the relationship between positive and negative pick-up ions and generated ion-cyclotron waves. He showed pick-up ion densities could be estimated from Galileo Plasma Wave Spectrometer data. These densities can be used to constrain mass loading estimates of the Europa plasma torus. Desai suggested that because this method probes the minority ion species, it might be used to investigate the possibility of a salt-rich subsurface ocean of Europa.

Moving from Jupiter to Saturn and drawing parallels to Kinrade's talk in the morning session, Greg Hunt (Leicester) particle perturbations of Saturn close to the planetary period, which are different in the northern and southern hemispheres. The present model of two separate but interacting rotating current systems, one in each hemisphere (Hunt et al. 2015), has been successfully applied to reflect Cassini magnetometer and radio data. Hunt examined the local time effects of the two systems, showing that current profiles are similar between PPO-dependent and independent currents. The Saturn Kilometric Radiation is a measure of field aligned current (FAC) strength and shows intensification at dawn. The model cannot account for this intensification; it predicts that the upward and downward FACs should cancel. spoke about planetary period oscillations (PPO) - the observation of field and
Staying at Saturn, Sam Taylor (MSSL) showed the results of an investigation that used Cassini Electron Spectrometer data to examine the photoelectron population around Enceladus, a moon known for its icy plumes. He demonstrated that there are primary and secondary energy peaks in the electron spectra and that, in the plumes, the ratio of the primary to secondary peak increases. This will allow the future analysis of ionization rates and distribution of neutral species around Enceladus, particularly in its plumes. Taylor also showed that modelling of photoelectron production and comparison with Cassini observations can provide an independent measure of the spacecraft potential.

The first talk of the conference dealt with phenomena which are very close to Earth, and the final talk was set at the furthest distance from us: Lars Mejnertsen (Imperial) demonstrated the power of global simulation and comparative magnetospherics in the far reaches of the solar system, where observations are sparse. At Neptune, the only data available are from the Voyager 2 flyby. Using a 3D resistive MHD model of Neptune's magnetosphere, he simulated the Voyager 2 flyby. During the flyby, the simulation showed evidence of dayside magnetopause reconnection as the planet rotated to face the solar wind pole-on. This indicated that planetary rotation modulates reconnection at Neptune.

\section{Summary}

All in all, the day was packed with oral presentations after a huge number of submitted abstracts. Although this was the first year that a registration fee was levied, The day was packed ance and the programme was with presentations after a huge number well received by attendees, with many people commenting on a day of exciting new developments in MIST science. To accompany the talks, 22 posters were presented. Although none of the talks during the main session dealt with the inner planets, posters were presented on Mercury and Mars, as well as on Earth and the outer planets.

MIST Council would like to thank the RAS for hosting the meeting, everyone who submitted an abstract, everyone who attended and everyone who said hello to us in the Walkers of St James afterwards, where scientific discussion continued long into the evening. $\bullet$

\section{AUTHORS \\ John Coxon, Dept of Physics and}

Astronomy, University of Southampton,

UK;work@johncoxon.co.uk.

Jasmine Sandhu, Dept of Physics

and Astronomy, University of Leicester,

UK. Nadine Kalmoni, UCL Mullard

$\begin{array}{ll}\text { Space Science Laboratory, Dorking, UK. } & \text { REFERENCES } \\ \text { Rebecca Gray, Joe Kinrade, Physics } & \text { Ashrafi M 2007 Astron. \& Geophys. } \\ \text { Dept, Lancaster University, UK. Ian } & \mathbf{4 8 4 . 3 5} \\ \text { McCrea, STFC Rutherford Appleton } & \text { Carter J A et al. 2015 J. Geophys. Res. } \\ \text { Laboratory for Atmospheric Science } & \text { Space Physics 120 2967 } \\ \text { and NERC National Centre for } & \text { Chen CH K et al. 2014 Astrophys. J. } \\ \text { Atmospheric Science, UK. } & \text { Letters 789(1) L8 }\end{array}$

Atmospheric Science, UK
Dods J et al. 2015 J. Geophys. Res. Space

Physics 1207774

Elsden T \& Wright AN $2015 \mathrm{~J}$.

Geophys. Res. Space Physics 120166

Forsyth C et al. $2015 \mathrm{~J}$. Geophys. Res.

Space Physics 12010592

Hunt G J et al. 2015 J. Geophys. Res.
Space Physics 1207552

Kalmoni NME et al. $2015 \mathrm{~J}$. Geophys. Res. Space Physics $\mathbf{1 2 0} 8503$ Usoskin IG et al. 2016 Solar Physics doi:10.1007/s11207-015-0838-1 